\title{
Statement of Simulation Experiments on the Models of Formation of the Advertising Strategies of Pharmaceutical Enterprises
}

\author{
Zoia Sokolovska \\ Department of Economical Cybernetics \\ and Informatics Technologies \\ Odessa National Polytechnic University \\ Odessa, Ukraine \\ nadin_zs@te.net.ua
}

\author{
Oksana Klepikova \\ Department of Economical Cybernetics \\ and Informatics Technologies \\ Odessa National Polytechnic University \\ Odessa, Ukraine \\ klepoa@ukr.net
}

\author{
Iryna Kapustyan \\ Department of Economical Cybernetics \\ and Informatics Technologies \\ Odessa National Polytechnic University \\ Odessa, Ukraine \\ kapustyanirene@gmail.com
}

\begin{abstract}
The questions of set-up simulation experiments on models of complex economic systems are considered. The main modern trends of the pharmaceutical industry are identified based on review of existing research in the field of experiments planning. The hypothesis of the feasibility of stating and conducting simulation experiments on the developed models-simulators is proposed for the enterprises of pharmaceutical industry of Ukraine in the process of formation their advertising strategies. The following aspects of experiments are discussed: determining the range of significant input factors; assessing models adequacy; processing and analysis of the final results; establishing the degree of their sensitivity to specific factor influences. The features of different types of experiments are determined, supported by the software implementation of the models on the platform of the system of multilevel simulation modeling AnyLogic. The authors emphasize effectiveness of experimental support for making managerial decisions regarding the positioning enterprises in the conditions of the entropy of pharmaceutical market. The research findings are tested on the materials of the leading pharmaceutical enterprises; they prove the possibility of adapting the models to the specifics of certain enterprises in the industry.
\end{abstract}

Keywords-simulation modeling, experiment, statement of experiment, pharmaceutical enterprise, modeling advertising strategies.

\section{INTRODUCTION}

Simulation models are powerful tools for studying real processes in complex economic systems. The advantages of this direction of simulation are: the ability to reproduce the deployment of processes in dynamics in real or virtual time scale; simulation of the effects of various stochastic factors; the availability of tools for creating modular and open model application-simulators for working out management decisions on the user-defined perspectives.

Construction and operation of models is closely linked with the statement of simulation experiments. This is a multistep procedure from the formulation of the problem to the processing of the results. In the range of questions about statement of experiments are included: assessing the suitability (adequacy) of the model; definition of complex of the most important factors to be determined for the model and further research; methods for evaluating the experimental alternatives; determination of the moments of stopping experiments, etc.

Many experts, working in the field of simulation modeling, pay attention to the problem of stating experiments, but for each new model application, it should be solved individually. Objectively, this is due to the specifics of real research objects, the goals (tasks) of experiments, the flexibility of algorithmic and programmatic implementation of simulation models.

According to the above, in this research the hypothesis of theoretical and applied expediency of statement and realization of simulation experiments on modelsimulators, designed for enterprises of the pharmaceutical industry of Ukraine in the process of forming their advertising strategies, is put forward.

The study proves:

- the definition of complex of significant parameters and estimation of the sensitivity of the developed models to the influence of these parameters in conditions of entropy of the advertising environment;

- the adequacy of developed model-simulators, worked out on materials of the leading enterprises of the Ukraine pharmaceutical industry;

- the effectiveness of making managerial decisions regarding the promotion of pharmaceutical products based on the results of simulation experiments.

\section{Analysis Of ReCENT ReSEARCh AND PUblications}

Since the creation of simulation modeling the questions of planning simulation experiments were at the center of researchers' attention and are reflected in many special literary sources. Innovations in this field are regularly presented in such professional international forums as Winter Simulation Conference (WSC) [1], International System Dynamics Conference [2], ASIM (German-speaking community of simulation modeling) [3], IMMOD ("Simulation modeling. Theory and practice") [4], European congress EUROSIM [5]; in webinars of one of the world's leading corporations in this 
industry - The AnyLogic Company [6]. Let us dwell briefly on some of the main trends.

The selection of the most significant input factors, that it is expedient to use in the simulation model, is a complex task, which determines the solution of the problem of "optimal complexity" and the overall simulation efficiency. Reference [7] is interest from the circle of recent publications. It is aimed at practical recommendations for the identification of important factors with less number of simulations in the process of designing simulation experiments. Latin designs of hypercubes, intended for nonlinear reactions, usually associated with simulation models, are presented along with the classical experimental approaches (complete, fractional, mixed factorial experiment).

Analysis of simulation results is an integral part of the planning of simulation experiments on models. There are various analytical approaches. They also went through a certain evolution during the active development of simulation methods. Reference [8] gives a careful overview of the methods of statistical analysis of simulation results. The authors focus on the main concepts and methodologies that have shaped the industry, as well as on the stages of its formation.

Such aspect of planning simulation researches, as simulation optimization, is closely related to the above. Reference [9] pays attention to the methods of ranking and selection, the stochastic approximation and gradient estimation, the method of analysis of the response surface and the average sampling approximation. A positive feature of the work is the discussion of the interaction of research with the specific practice of use. The development of appropriate software for optimizing the process of carrying out experiments was included in the circle of questions.

Reference [10] also develops this topic. The authors emphasize on such aspect of experiment planning as reducing dispersion due to dependency structures and efficient modeling of rare events. Both retrospective research results and recent innovations are presented.

Assessment of the degree of uncertainty and errors, inherent in the processes of their use compared with the actual objects of research, is one of the important issues of the analysis of simulation models adequacy. Although any model is a simplified reproduction of reality, the feasibility of its exploitation in the decision-making process should be established from the viewpoint of the existing uncertainty - the issue is ambiguous and poorly studied at the current stage of simulation development. Reference [11] offers a structure for uncertainty analysis for simulation models. The authors determined the sequence of steps, which should be performed during the uncertainty analysis of simulation models. Also discusses the structure, in which entropy is used as a possible measure of uncertainty of costs. The structure is proposed as a regulator of managerial decisions with a view of possible errors and inaccuracies of modeling results.
Significant contribution to the development of methodology for planning simulation experiments was made by the works of V. V. Devyatkov's science school. The generalization of many years of research has been reflected in the monograph [12]. In this work the author pays close attention to the stages of planning experiments and, in particular, to assessing the adequacy of models to real objects and processes of research.

In domestic Ukrainian practice, the issues of experiment planning are mainly considered in the construction of models of technical systems. In particular, references [13] provides further development of the Monte-Carlo method and pays attention to improving its adaptation for simulation testing in order to evaluate the trouble-free operation of technical systems.

Despite the research of technical systems, the obtained mathematical results can be used in constructing and running the simulation experiments on the systems and processes of economic content, including the pharmaceutical industry. A similar example of possibility of using the results obtained is the references [14].

In the field of simulation research of the pharmaceutical market and its components, it is worthwhile to highlight the development of consulting firms Bayser (Bayser Consulting) [15] and Sterling (Sterling Simulation) [16], which are aimed at studying the behavior of consumers during the formation of marketing strategies by large pharmaceutical companies. An agent approach was used when models are constructing. Patients, doctors, sales representatives of pharmaceutical companies, pharmaceuticals were acted as agents in both cases. Experiment planning was carried out in accordance with the capabilities of the software platform for models implementation - the system of multilevel simulation modeling AnyLogic.

At the same time, it should be noted separately, that in the given subject area today there are only isolated solutions using the simulation apparatus, which mostly relate to the production and sales sector. Focusing on the processes of statement experiments on simulation model is determined by the dynamics and high degree of entropy of the pharmaceutical market; by the need to take into account many factors of a stochastic nature, the composition of which varies depending on set by the experimenter goals.

\section{PRESENTATION OF KEY RESEARCH FINDINGS}

Within the framework of research conducted for leading pharmaceutical companies of Ukraine (PJSC "Farmak", "Arterium" Corporation, pharmaceutical company "Darnitsa"), a complex of simulation models of advertising activity of a typical pharmaceutical enterprise was developed. The software platform for implementing models is the AnyLogic system that supports the multiapproach paradigm of simulation modeling.

The module for the advertising strategy formation for the sale of original and generic medicines based on the simulation of consumer behavior, depending on the 
situation on the market of pharmaceutical products, is one of the components of the complex. Its allows to adjust the necessary amount of expenses for advertising tools; to determine the reasonable cost of pharmaceutical products; to optimize the effectiveness of marketing costs; to maximize revenue from advertising campaigns and the number of regular customers; to calculate the sales in kind, and also monitor changes in user preferences; respond in time to increase or decrease the demand for a certain category of medicines.

Advertising activities of a typical pharmaceutical enterprise are characterized by a significant number of parameters. As a result of the analysis, the following most significant factors of the simulation model were highlighted: product price; demand for products; investment in advertising activities; medicines development costs; advertising effectiveness.

The main results of the system functioning are sales revenue, sales volumes, the number of potential and regular buyers, the number of developed medicines. From the point of view of making management decisions, when parameters and functions were subject to change, the most effective were the sales revenue and the number of regular buyers.

Based on the analysis of the available data and the possibilities of influencing the input parameters of the model, it was decided to use the Nerlove-Arrow advertising model ( $\mathrm{N}-\mathrm{A}$ model) to describe the process of informing the target audience. This N-A model has the form of a 1st order differential equation:

$$
A(t)=b \cdot q(t)-k \cdot A
$$

where $A(t)$ - product awareness (number of informed people about the product in period $\mathrm{t}) ; q(t)$ - advertising activity (advertising costs in per. t) $; b$ - advertising effectiveness (advertising source rating); $k$ - speed of forgetting information. In a simulation model, this ratio is defined as a random variable from $10 \%$ to $80 \%$, due to the Ebingaus forgetting curve.

The results obtained using the simulation model should have the required accuracy and reliability. The reliability of the simulation results suggests that the simulation model correspond to some specific requirements, which meet the goals of the simulation. Assessment of the properties and quality of the model involves an assessment of the model adequacy, the study of the model sensitivity, etc.

It is customary to determine the model adequacy to determine the degree of simulation compliance with a real object and the goals of the research. One of the most common ways to justify the model adequacy is to use the methods of mathematical statistics. The essence of these methods is to test the hypothesis put forward on the basis of some statistical criteria.

The criteria of agreement (or criteria of conformity) are special statistical indicators used to test the hypothesis of conformity of the empirical distribution to the theoretical distribution law. The modified KolmogorovSmirnov, Pearson (chi-square), Kramer-Mises-Smirnov (omega-square) criteria for the case of two samples are using the most widely.

Most agreement criteria are based on the use of empirical frequency deviations from theoretical ones. It is obvious that the theoretical distribution better corresponds to the empirical distribution (or describes it) when these deviations are the smallest.

The analysis of the forecast adequacy of the share of exports in the total sales of PJSC "Farmak" based on quarterly sales data [17] in the period from 2015 till 2018 was conducted using two-sample Kolmogorov-Smirnov agreement criterion.

The null hypothesis $\mathrm{H}_{0}$ : "the differences between the two distributions are not significant". Experimental criterion value $\lambda_{\exp }$ determined by the formula:

$$
\lambda_{\exp }=d_{\max } \cdot \sqrt{\frac{n_{t} \cdot n_{e}}{n_{t}+n_{e}}}
$$

where $d_{\max }$ - maximum absolute difference between empirical and theoretical cumulative frequencies, $d_{\max }$ $=0,0168 ; n_{t}$ and $n_{e}-$ respectively, volumes of theoretical and empirical samples,$n_{t}=n_{e}=16$.

According to the formula (2) $\lambda_{\exp }=0,048$. At the level of significance $a=0,05$ critical criterion value $\lambda_{\text {cr }}(0,05)=1,358$. Thus, $\lambda_{\text {exp }}=0,048<\lambda_{\text {cr }}=1,358$. Therefore, the null hypothesis is accepted, the theoretical values of the share of exports differ from empirical values only slightly.

Using the two-sample Pearson agreement criterion $\chi 2$ provides for dividing the range of sampling variation into intervals and determining the number of observations (frequency) for each of the intervals. The number of intervals depends on the sampling size. The analysis of the prediction adequacy of the number of packages quarterly sold by "Arterium" Corporation [18] in the period from 2015 till 2018 was conducted using this criterion.

In the Pearson criterion, the measure of the discrepancy between the theoretical and empirical distributions is the weighted sum of squared deviations:

$$
\chi_{\exp }^{2}=n_{e} n_{t} \sum_{i=1}^{k} \frac{1}{m_{i}^{t} \cdot m_{i}^{e}}\left(\frac{m_{i}^{t}}{n_{t}}-\frac{m_{i}^{e}}{n_{e}}\right)^{2}
$$

where $k$ - number of intervals for splitting the parameter values, $k=5 ; n_{t}$ and $n_{e}$ - respectively, the number of theoretical and empirical observations, $n_{t}=n_{e}=16 ; m_{t}$ and $m_{e}-$ respectively, relative frequencies of theoretical and empirical distribution;

The null hypothesis $\mathrm{H}_{0}$ : "the differences between the two distributions are not significant". The null hypothesis is accepted if the observed value of the criterion does not exceed its critical value. 
According to the formula (3) $\chi_{\text {exp }}^{2}=0,845$. Table value of criterion at significance level $a=0,05$ is $\chi_{1-a, k-1}^{2}=9,5$. Thus, $\chi_{\text {exp }}^{2}=0,845<\chi_{0.95,4}^{2}=9,5$. Therefore, the null hypothesis is accepted, theoretical values of the number of sold packages are slightly different from empirical values.

The two-sample Kramer-Mises-Smirnov agreement criterion $\omega^{2}$ (omega-square) is based on calculation the sum of squared differences between the cumulative frequency (empirical distribution function) and the theoretical distribution function. Statistics criteria can be calculated by the formula:

$$
\omega_{\exp }^{2}=\frac{n_{e} \sum_{i=1}^{n_{e}}\left(r_{i}-i\right)^{2}+n_{t} \sum_{j=1}^{n_{t}}\left(s_{j}-j\right)^{2}}{n_{e} n_{t}\left(n_{e}+n_{t}\right)}-\frac{4 n_{e} n_{t}-1}{6\left(n_{e}+n_{t}\right)}
$$

where $n_{t}$ и $n_{e}$ - the number of theoretical and empirical observations, respectively, $n_{t}=n_{e}=16 ; r_{i}-$ the rank of the element in the empirical sample; $s_{j}-$ the rank of the element in the theoretical sample.

The analysis of the forecast adequacy of the volume of quarterly sales by the pharmaceutical company "Darnitsa" [19] in the period from 2015 till 2018 was conducted using the omega-square criterion. The null hypothesis $\mathrm{H}_{0}$ : "distributions are homogeneous". The null hypothesis is accepted if the observed value of the criterion does not exceed its critical value.

According to the formula (4) $\omega_{\text {exp }}^{2}=0,328$. Tabular value of the criterion at the level of significance $a=0,05$ $\omega_{1-a}^{2}=0,461$. Thus, $\omega_{\text {exp }}^{2}=0,328<\omega^{2}{ }_{0,95}=0,461$. Therefore, the null hypothesis is accepted, the theoretical distribution of sales volumes corresponds to the empirical distribution.

The specificity of the pharmaceutical industry is the research and analysis of data not only in general, but also by region of the country. For making management decisions regarding the approval of a particular marketing strategy, it is important to understand which sets of factors with which values are weighty and significant for the respective regions.

To determine the difference significance of two or more sets (combinations) of factors on the regions, it is advisable to apply the $Q$ criterion Cochran - a nonparametric criterion whose response is a dichotomous variable (it takes two values - $0 / 1$; yes/no). Criterion hypotheses: $\mathrm{H}_{0}$ - "there are no significant differences in the effects on the regions", $\mathrm{H}_{1}-$ "there are significant differences in the effects on the regions". Statistics of $Q$ criterion Cochren is determined by the formula:

$$
Q=k(k-1) \frac{\sum_{j=1}^{k}\left(X_{. j}-\frac{N}{k}\right)^{2}}{\sum_{i=1}^{m} X_{i .}\left(k-X_{i .}\right)}
$$

where $k$ - number of impacts; $X_{. j}$ - sum of positive responses for j-th impact; $m$ - number of regions; $X_{i \text {. }}$ - sum of positive responses for the i-th region; $N$ - total number of observations.
The null hypothesis is rejected if the criterion statistics falls into the critical region $Q>\chi_{1-a, k-1}^{2}$, where $\chi_{1-a, k-1}^{2}$ chi-square distribution quantile with $k-1$ degrees of freedom and significance level $a$.

The study of the effects of three averaged groups of parameters on 5 regions of Ukraine - Central, Southern, Northern, Eastern and Western - was conducted:

- I parameter group: reduced price for products; average investment in advertising; increased number of advertising sources.

- II parameter group: increased product prices; reduced investment in advertising; average number of sources of advertising.

- III parameter group: average price of products; increased investment in advertising; reduced number of sources of advertising.

Depending on the group of parameters, used in a typical pharmaceutical enterprise, the level of sales volume changes, respectively, the niche occupied by the market changes, too. Experimental statistics of the Q criterion were calculated based on the obtained data on the achievement / non-achievement by country regions of a given level of sales, taking into account the application of the corresponding sets of parameters: $Q_{\exp }=8.40$. When $a=0,995$ quantile $\chi_{1-a, k-1}^{2}=10,60 . \quad Q_{\text {exp }}<\chi_{1-a, k-1}^{2}$, therefore, the null hypothesis is accepted. Different in character sets of parameters in practice do not have a significant difference in the impact on the regions of the country.

However, in practice other groups of parameters are possible:

- IV parameter group: reduced price for products; reduced investment in advertising; the average number of sources of advertising.

- V parameter group: increased product prices; average investment in advertising; increased number of advertising sources.

For five sets of parameters, affecting the regions of Ukraine, experimental statistics of the $\mathrm{Q}$ criterion: $Q_{\text {exp }}=21.43$. When $a=0,995$ quantile $\chi_{1-a, k-1}^{2}=14,86$. $Q_{\text {exp }}>\chi_{1-a, k-1}^{2}$, therefore the null hypothesis is rejected. There are significant differences in the effect of parameter sets on country regions.

The analysis of the models adequacy was based on conducting a large number of experiments. AnyLogic software platform supports various types of simulation experiments: from simple (standard) to non-standard experiment with fully customizable script. Besides the standard experiment, the following experiments were carried out on the developed models:

- Optimization (solves the task of optimizing a given target function with the set restrictions on the values of parameters and variables model). The optimal values of input parameters of the 
investigated advertising companies with the target function of maximizing the level of incomes are determined using the Optimization experiment.

- Variation of parameters (implements a series of model runs with variations of one or several parameters, with the possibility of using replication). The analysis of the income levels' dynamics at different values of the production cost carried out by conducting the experiment Variation of parameters.

- Comparison of runs (allows interactively to set different values of parameters; visually compares the results of "runs" in the scalar form, as well as in the form of data sets). Regular_buyers, Income and Product_cost used as parameters. The experiment clearly demonstrated how the behavior of these variables depends on each other.

- Sensitivity analysis (execution of model runs with variation of values of one parameters and determination of its effects on the final modeling results). The sensitivity of the model to the changes of certain significant factors was investigated using this type of experiments. The greatest degree of sensitivity of the system has been experimentally proved to such factors as the costs of advertising and the cost of pharmaceutical products.

- Calibration. The values of the model parameters were found with the help of the optimizer, in which the results of the simulation most closely correspond to the given data. Data was given both in scalar form and in the form of data sets. The visualization of the progress of calibration and correspondence of the results to each given criterion was performed.

The process of model calibration, along with the results of parametric experiments and sensitivity estimation, allowed formulating an idea of the general degree of models uncertainty and their generalizations in relation to the actual simulated processes.

The prognostic calculations of the main components of the advertising strategies of pharmaceutical companies were carried out using the given complex of simulation experiments, performed on the developed models. The obtained results proved the predictive accuracy of the dynamics evaluation of the investigated indicators of the pharmaceutical companies functioning (on the example of leading enterprises of the industry). Experiments have also proven the ability to adapt models to the specifics of concrete enterprises in the industry.

\section{CONCLUSIONS AND RECOMMENDATIONS}

The obtained results testify to the sufficient adequacy of models for real processes of formation and implementation of advertising strategies at the level of pharmaceutical enterprises. This contributes to achievement of the main goal - the creation of effective models of simulators for the preliminary experimental working out strategic and operational management decisions on them regarding the promotion of pharmaceutical products by means of advertising. Available options for customizing models for different types of experiments provide operation of the model complex in the process of monitoring the passage of advertising companies.

Migration of simulation computations to the cloud will contribute to the improvement of the formulation and implementation of experiments. As an experimental base, the cloud can be considered in the following directions:

- Ensuring and expanding computing power. This is especially true for "multi-run" types of experiments: Monte Carlo, Variation of parameters, Optimization.

- Support for the universal environment of work with the model: work with versions of the model; task of input parameters; planning and implementation of complex experiments; review, analyze and export results; providing animation of a remotely implemented model and implementing its interactivity (interactive model management while performing experiments).

Further research is planned to focus on expanding the experimental base through typical and non-standard plans of simulation experiments, adapted to the specifics of the industry. The evaluation effectiveness of model experiments will be deepened, in particular, through the use of spectral analysis.

\section{REFERENCES}

[1] Winter Simulation Conference website, 2018. [Online]. Available: http://meetings2.informs.org/wordpress/ wsc2018/. Accessed on: Aug. 27, 2019.

[2] The 36th International Conference of the System Dynamics Society website, 2018. [Online]. Available: https://www.systemdyna-mics.org/past-conference-2018. Accessed on: Aug. 27, 2019.

[3] Arbeitsgemeinschaft Simulation website, 2019. [Online]. Available: https://www.asim-gi.org/asim/. Accessed on: Aug. 27, 2019.

[4] Eighth All-Russian Scientific and Practical Conference "Simulation modeling. Theory and practice" IMMOD-2017, 2017. [Online]. Available: http://simulation.su/ static/ru-immod2017.html. Accessed on: Aug. 27, 2019.

[5] The Federation of European Simulation Societies website, 2019. [Online]. Available: https://www.eurosim.info/ eurosim/. Accessed on: Aug. 27, 2019.

[6] AnyLogic Company website, 2019. [Online]. Available: http://www.anylogic.com/. Accessed on: Aug. 27, 2019.

[7] M. Law, "A tutorial on design of experiments for simulation modeling", in Proc. WSC'17, Las Vegas, Dec. 2017, pp.550-564.

[8] Alexopoulos, and W. D. Kelton, "A concise history of simulation output analysis", in Proc. WSC'17, Las Vegas, Dec. 2017, pp.116130 .

[9] M. C. Fu, and S. G. Henderson, "History of seeking better solutions, AKA simulation optimization", in Proc. WSC'17, Las Vegas, Dec. 2017, pp.131-157.

[10] R. Barton, M. K. Nakayama, and L. Schruben, "History of improving statistical efficiency", in Proc. WSC'17, Las Vegas, Dec. 2017, pp.158-180.

[11] P. G. Scheidegger, T. F. Pereira, and A. Banerjee, "Uncertainty quantification in simulation models: a proposed framework and application through case study", in Proc. WSC'18, Las Vegas, Dec. 2018, pp.1599-1610. 
[12] V. V. Devyatkov, "The Development of Methodology of Simulation Studies of Complex Economic Systems", Ph.D. diss., Financial University under the Government of the Russian Federation, 2014.

[13] S. V. Egorov, and T. U. Shkvarnitskaya, "Investigation of the method of determining the infallibility of technical systems using test simulation", Technology audit and production reserves, Vol. 2/2(28), pp. 25-29, 2016.

[14] P. S. Kosobutskiy, M. V. Kuzminikh, and A. Morgulis, "About probabilistic-statistical estimation of a sample of random values of experimental data", in Proc. MODS'18, Chernihiv, Jun. 2018, pp. 219-222.
[15] Bayser Consulting. Modeling of a Pharmaceutical Product Launch, 2019. [Online]. Available: https://www.any logic.com/modelingof-a-pharmaceutical-product-launch/. Accessed on: Aug. 25, 2019.

[16] Sterling Simulation. A Pharmaceutical Company Decides on a Marketing Strategy Using Agent-Based Modeling, 2019. [Online]. Available: https://www.anylogic.com/a-phar maceutical-companyused-agent-based-modeling-to-decide-on-a-marketing-strategy/. Accessed on: Aug. 25, 2019.

[17] OJSC "Farmak" website, 2019. [Online]. Available: https://farmak.ua/en/. Accessed on: Jul. 17, 2019.

[18] "Arterium" Corporation website, 2019. [Online]. Available: http://www.arterium.ua/en. Accessed on: Jul. 17, 2019.

[19] Pharmaceutical company "Darnitsa" website, 2019. [Online]. Available: https://www.darnitsa.ua/en/. Accessed on: Jul. 17, 2019. 